\title{
Throughput Maximization in Wireless Powered Communication Networks with Energy Saving
}

\author{
Rui Wang, D. Richard Brown III \\ Dept. of Electrical and Computer Eng. \\ Worcester Polytechnic Institute \\ 100 Institute Rd, Worcester, MA 01609 \\ Email: $\{$ rwang,drb\}@wpi.edu
}

\begin{abstract}
This paper considers a time division multiple access scenario where a wireless access point transmits to a group of users which harvest the energy and then use this energy to transmit back to the access point. Past approaches have found the optimal time allocation to maximize sum throughput under the assumption that the users must use all of their harvested power in each block of the "harvest-then-transmit" protocol. This paper considers optimal time and energy allocation to maximize the sum throughput for the case when the nodes can save energy for later blocks. To maximize the sum throughput over a finite horizon, the initial optimization problem is separated into two sub-problems and finally can be formulated into a standard box-constrained optimization problem, which can be solved efficiently. A tight upper bound is derived by relaxing the energy harvesting causality. Simulation results are also provided to demonstrate the "harvest-then-transmit" protocol with energy saving provides improved sum throughput increasing with the number of transmission blocks.
\end{abstract}

Index Terms-wireless power transfer, energy harvesting, sum throughput maximization

\section{INTRODUCTION}

Prolonging the lifetime of battery powered devices in wireless networks is an important problem [1]. Replacing or recharging batteries may be inconvenient (e.g., for a sensor network with massive distributed sensor nodes), dangerous (e.g., for devices positioned in toxic environments), or even impossible (e.g., for the medical sensors implanted inside human bodies). To overcome such situations, energy harvesting has become an attractive approach with the potential of extending the lifetime of these devices. Energy harvesting nodes have the ability to recharge their batteries from their surrounding environment by using solar, heat, vibration, or other energy sources [2], [3].

Recently, wireless power transfer (WPT) using radio frequency signals is attracting attention as a viable approach to the energy harvesting problem. One approach to WPT is to harvest energy from ambient radio signals, e.g., TV broadcast signals [4]. Another approach to WPT is to use a dedicated power transmitter such as in passive radio frequency identification (RFID) systems [5], [6]. WPT systems can simultaneously convey energy and information on the wireless signals [7]-[9] and the inherent tradeoff between information rate and power transfer efficiency has been recently characterized [10], [11]. For the energy harvesting case, maximizing a time-average utility function over infinite time blocks (infinite horizon) is considered in [12]. In [13], the authors consider the problem of maximizing the throughput of a transmitter sending data over a time-varying channel within finite time blocks (finite horizon) under a total energy constraint. In [14], an explicit threshold policy is derived for energy harvesting sensors to maximize the utility obtained over a finite horizon.

In this paper, we consider WPT system called a "wireless powered communication network" (WPCN). A WPCN is a network in which wireless devices are powered only by WPT [15]. The WPCN model considered in this paper is the same as in [15] and is shown in Fig. 1, where one hybrid access point (H-AP) with an effectively unlimited power supply coordinates the wireless energy/information transmissions to/from a set of distributed users. Each user is equipped with an energy storage device and thus can harvest and store the wireless energy broadcasted by the H-AP in the downlink. The users transmit their independent information using their individually harvested energy to the H-AP in the uplink. In [15], a block transmission model was considered where it was assumed that users harvest energy during a downlink transmission the first part of the block and then each user uses all of their harvested energy during an uplink transmission later in that block. In other words, users do not save energy for later blocks.

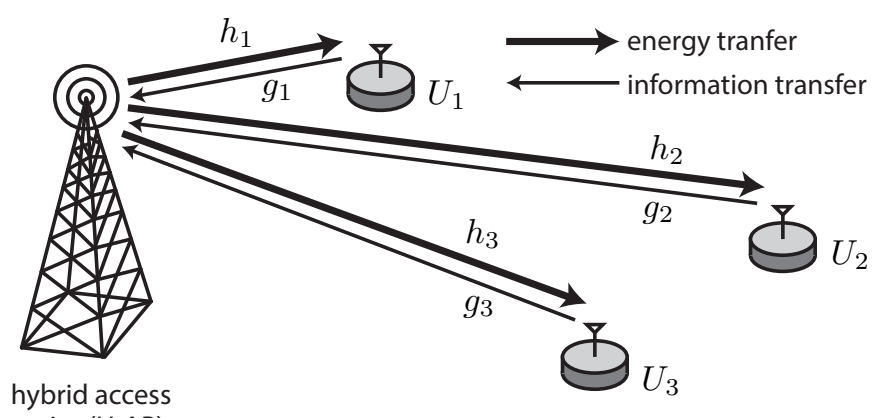

point (H-AP)

Fig. 1. A wireless powered communication network (WPCN).

The primary contribution in this paper is a generalization of the system considered in [15] where the users can save energy harvested in the current block for wireless information transmission (WIT) in later blocks. We consider the problem of maximizing the sum throughput over a finite 
horizon with energy saving. The analysis assumes an "oracle" provides knowledge of the channel states for all blocks prior to the commencement of the first block. Hence, the results developed in this paper can be considered an upper bound for finite-horizon energy saving schemes with causal channel knowledge. The initial optimization problem is separated into two sub-problems: (i) calculating the optimal time allocation by fixing energy allocation and (ii) calculating the optimal time allocation and energy allocation of downlink WET. The former is a convex optimization problem, which gives us a closed-form relation between the time allocation of donwlink WET and uplink WIT and the latter can be formulated as a standard box-constrained nonlinear programming problem, which can be solved efficiently using the trust-region-reflective algorithm [16], [17]. An upper bound with low computational complexity is provided by relaxing the energy harvesting causality, which give us a water-filling typed solution. Simulation results are also provided to demonstrate the "harvestthen-transmit" protocol with energy saving provides improved sum throughput increasing with the number of transmission blocks. The resulting gains are somewhat modest, however, and require significant computation as well as "oracle" channel estimates. Hence these results show that the original "harvestthen-transmit" protocol without energy saving is a practical strategy offering good performance for WPCN.

\section{SYSTEM MODEL}

The network adopts a harvest-then-transmit protocol as shown in Fig.2. In each block, the first $\tau_{0} T$ amount of time, with $\tau_{0} \in[0,1]$, is assigned to the downlink for the H-AP to broadcast wireless energy to all users, while the remaining time in the same block is assigned to the uplink for transmitting their independent information to the H-AP. We assume there are $K$ users in total and the amount of time assigned to user $U_{i}$ is denoted by $\tau_{i} T, \tau_{i} \in[0,1], \forall i \in \mathcal{I}$, where $\mathcal{I}:=\{1, \cdots, K\}$ is the set of the user indices. We have

$$
\sum_{i=0}^{K} \tau_{i} \leq 1
$$

since $\tau_{i}, \forall i \in \overline{\mathcal{I}}:=\{0\} \cup \mathcal{I}$, represent the allocated time portions in each block. To simplify analysis, we assume normalized unit time $T=1$.

If $P_{A}$ denotes the transmit power at the $\mathrm{H}-\mathrm{AP}$, the amount of energy harvested by each user in the downlink can be expressed as $E_{i}=\zeta_{i} P_{A} h_{i} \tau_{0}, \forall i \in \mathcal{I}$, where $h_{i}$ denotes the channel power gain of the $i$ th downlink channel and $\zeta_{i} \in(0,1)$ is the energy harvesting efficiency at $i$ th receiver. For convenience, it is assumed that $\zeta_{j}=\zeta_{k}=\zeta, \forall j, k \in \mathcal{I}$ for the remainder of this paper.

After the users replenish their energy during the downlink phase, in the subsequent uplink phase they transmit independent information to the H-AP in their allocated time slots. Instead of using all the energy harvested from the H-AP during current block, we assume that each user can save their energy for future blocks. To distinguish each block, we use superscript

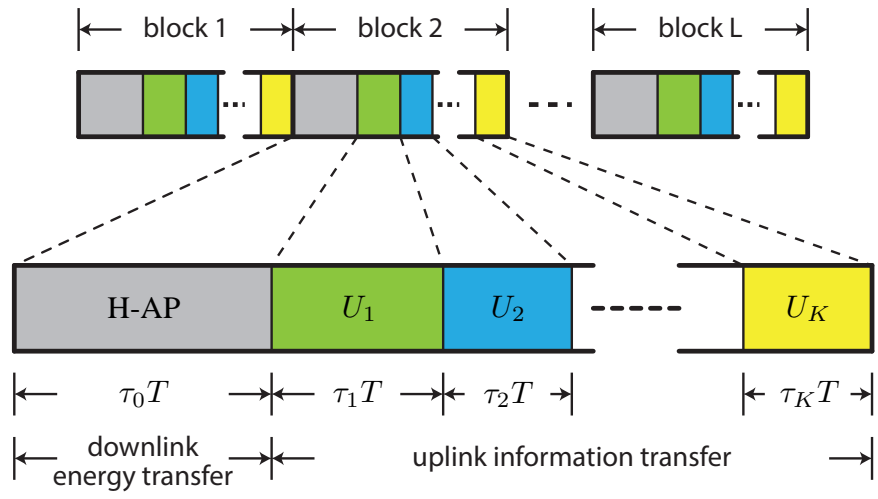

Fig. 2. Harvest-then-transmit protocol and block structure.

$(\ell)$ to denote the $\ell$ th transmission block. Thus, the energy harvested by user $U_{i}$ in $\ell$ th block can be written as

$$
E_{i}^{(\ell)}=\zeta P_{A} h_{i}^{(\ell)} \tau_{0}^{(\ell)}=\beta_{i}^{(\ell)} \tau_{0}^{(\ell)} .
$$

If we use $W_{i}^{(\ell)}$ and $F_{i}^{(\ell)}$ to denote the energy available and consumed by user $U_{i}$ during the $\ell$ th transmission block, respectively, and consider a finite horizon, say $L$ transmission blocks in total, then the following relation holds

$$
W_{i}^{(\ell)}=\sum_{j=1}^{\ell} E_{i}^{(j)}-\sum_{j=1}^{\ell-1} F_{i}^{(j)}, \forall i \in \mathcal{I}, \forall \ell \in \mathcal{J}
$$

where $\mathcal{J}:=\{1, \cdots L\}$ is the set of transmission block indices. Additionally, the amount of energy consumed in each block can not exceed the current energy stored for each user, i.e.,

$$
F_{i}^{(\ell)} \leq W_{i}^{(\ell)}, \forall i \in \mathcal{I}, \forall \ell \in \mathcal{J} .
$$

This corresponds to an energy causality constraint.

To simplify our analysis, we introduce parameters $\alpha_{i}^{(\ell)} \in$ $[0,1], i \in \mathcal{I}, \ell \in \mathcal{J}$, where $\alpha_{i}^{(\ell)}$ denotes the energy proportion consumed by $i$ th user in $\ell$ th block. Hence, we can rewrite the energy relations given in (3) as

$$
F_{i}^{(\ell)}=\alpha_{i}^{(\ell)} W_{i}^{(\ell)}, \forall i \in \mathcal{I}, \forall \ell \in \mathcal{J}
$$

The achievable uplink throughput of $i$ th user in bits/Hz during $\ell$ th block can be expressed as

$$
\begin{aligned}
R_{i}^{(\ell)} & =\tau_{i}^{(\ell)} \log _{2}\left(1+\frac{g_{i}^{(\ell)} F_{i}^{(\ell)}}{\Gamma \sigma^{2} \tau_{i}^{(\ell)}}\right) \\
& =\tau_{i}^{(\ell)} \log _{2}\left(1+\gamma_{i}^{(\ell)} \frac{F_{i}^{(\ell)}}{\tau_{i}^{(\ell)}}\right)
\end{aligned}
$$

where $\sigma^{2}$ is the variance of the received noise at the $\mathrm{H}$ AP, $\Gamma$ is the signal-to-noise ratio gap from the additive white Gaussian noise channel capacity due to a practical modulation and coding scheme used and $g_{i}^{(\ell)}$ represents the channel power gain of the $i$ th uplink channel during $\ell$ th block. It is assumed that the channel reciprocity holds for the downlink WET and uplink WIT, i.e., $h_{i}^{(\ell)}=g_{i}^{(\ell)}, \forall i \in \mathcal{I}, \forall \ell \in \mathcal{J}$. Then, the 
sum throughput of $K$ users over $L$ transmission blocks can be written as

$$
R=\sum_{\ell=1}^{L} \sum_{i=1}^{K} R_{i}^{(\ell)}
$$

To facilitate the analysis, we define the time allocation vector for downlink WET $\boldsymbol{\tau}_{0}$, the time allocation vector for uplink WIT $\boldsymbol{\tau}$ and the energy-consumed proportion vector $\boldsymbol{\alpha}$, respectively, as

$$
\begin{aligned}
\boldsymbol{\tau}_{0} & :=\left[\begin{array}{lll}
\tau_{0}^{(1)} & \cdots & \tau_{0}^{(L)}
\end{array}\right]^{\mathrm{T}} \in \mathbb{R}^{L \times 1} \\
\boldsymbol{\tau} & :=\left[\begin{array}{lll}
\left(\boldsymbol{\tau}^{(1)}\right)^{\mathrm{T}} & \cdots & \left(\boldsymbol{\tau}^{(L)}\right)^{\mathrm{T}}
\end{array}\right]^{\mathrm{T}} \in \mathbb{R}^{K L \times 1} \\
\boldsymbol{\alpha} & :=\left[\begin{array}{lll}
\left(\boldsymbol{\alpha}^{(1)}\right)^{\mathrm{T}} & \cdots & \left(\boldsymbol{\alpha}^{(L)}\right)^{\mathrm{T}}
\end{array}\right]^{\mathrm{T}} \in \mathbb{R}^{K L \times 1}
\end{aligned}
$$

with

$$
\begin{aligned}
\boldsymbol{\tau}^{(\ell)} & :=\left[\begin{array}{lll}
\tau_{1}^{(\ell)} & \cdots & \tau_{K}^{(\ell)}
\end{array}\right]^{\mathrm{T}} \in \mathbb{R}^{K \times 1}, \forall \ell \in \mathcal{J} \\
\boldsymbol{\alpha}^{(\ell)} & :=\left[\begin{array}{lll}
\alpha_{1}^{(\ell)} & \cdots & \alpha_{K}^{(\ell)}
\end{array}\right]^{\mathrm{T}} \in \mathbb{R}^{K \times 1}, \forall \ell \in \mathcal{J} .
\end{aligned}
$$

Then, the sum throughput over $L$ transmission blocks in (6) can be expressed as a function with respect to $\left(\boldsymbol{\tau}_{0}, \boldsymbol{\tau}, \boldsymbol{\alpha}\right)$ and is denoted as $R\left(\boldsymbol{\tau}_{0}, \boldsymbol{\tau}, \boldsymbol{\alpha}\right)$. Our goal is to find the optimal time allocation vector of downlink WET $\tau_{0}$, the optimal time allocation vector of uplink WIT $\boldsymbol{\tau}$ and the energy-consumed proportion vector $\boldsymbol{\alpha}$ simultaneously to maximize the sum throughput over $L$ transmission blocks in (6). Mathematically, the sum throughput maximization problem is formulated as

Problem 1 (P1).

$$
\begin{array}{ll}
\max _{\left(\boldsymbol{\tau}_{0}, \boldsymbol{\tau}, \boldsymbol{\alpha}\right)} & R\left(\boldsymbol{\tau}_{0}, \boldsymbol{\tau}, \boldsymbol{\alpha}\right) \\
\text { s.t. } & \sum_{i=0}^{K} \tau_{i}^{(\ell)} \leq 1, \forall \ell \in \mathcal{J} \\
& \boldsymbol{\tau}_{0} \succeq \mathbf{0} \\
& \boldsymbol{\tau} \succeq \mathbf{0} \\
& \mathbf{0} \preceq \boldsymbol{\alpha} \preceq \mathbf{1} .
\end{array}
$$

We notice that $\mathbf{P 1}$ is a non-convex optimization problem since it's objective function contains non-convex terms. In the following section, we provide an algorithm to solve P1 by separating it to two sub-problems.

\section{Optimal Solutions of P1}

In this section, we provide the method of finding the optimal solution of P1. Instead of solving P1 directly, we first change $\mathbf{P 1}$ to an equivalent problem $\mathbf{P 2}$, then consider the problem $\mathbf{P 3}$ to find the optimal time allocation vectors of both downlink WET and uplink WIT by fixing the energy-consumed proportion vector. After solving the equations obtained from the KKT conditions of $\mathbf{P 3}$, we find a uniform relation between the optimal time allocation of downlink WET and the time allocation of uplink WIT. Thus, $\mathbf{P 2}$ can be transformed into a standard box-constrained optimization problem $\mathbf{P 4}$ by using this relation, which can be efficiently solved by the trustregion-reflective algorithm [16], [17].
First of all, it is straightforward to obtain the following lemma.

Proposition 1. The optimal time allocation $\left(\boldsymbol{\tau}_{0}^{\star}, \boldsymbol{\tau}^{\star}\right)$ of $\boldsymbol{P 1}$ must satisfy

$$
\sum_{i=0}^{K} \tau_{i}^{(\ell) \star}=1, \forall \ell \in \mathcal{J}
$$

The proof is omitted due to space limitations. From Proposition 1, we can obtain the equivalent optimization problem of P1 with equality constraints shown in (7), which is denoted as $\mathbf{P 2}$. Since $\mathbf{P 2}$ is also a non-convex problem, it is not easy to solve $\mathbf{P 2}$ directly. To overcome this, we first consider the problem of finding the optimal time allocation vectors of downlink and uplink $\left(\boldsymbol{\tau}_{0}^{\star}, \boldsymbol{\tau}^{\star}\right)$ to maximize the sum throughput over $L$ transmission blocks given a fixed energy-consumed proportion vector $\alpha$. Mathematically, the optimization problem can be formulated as

Problem 3 (P3).

$$
\begin{array}{ll}
\underset{\left(\boldsymbol{\tau}_{0}, \boldsymbol{\tau}\right)}{\max } & R\left(\boldsymbol{\tau}_{0}, \boldsymbol{\tau}, \boldsymbol{\alpha}\right) \\
\text { s.t. } & \sum_{i=0}^{K} \tau_{i}^{(\ell)}=1, \forall \ell \in \mathcal{J} \\
& \boldsymbol{\tau}_{0} \succeq \mathbf{0} \\
& \boldsymbol{\tau} \succeq \mathbf{0}
\end{array}
$$

where the parameters $\boldsymbol{\alpha}$ in $\mathbf{P 3}$ is fixed.

\section{Proposition 2. P3 is a convex optimization problem.}

The proof is omitted due to the space limitation. We know that the necessary Karush-Kuhn-Tucker (KKT) conditions of $\mathbf{P 3}$ are also sufficient and any local maximum solution of $\mathbf{P 3}$ is also a global maximum solution [18]. According to the harvested energy expression in (1) and the energy relations given in (2) and (4), we can obtain the relation between the consumed energy vector $\boldsymbol{F}_{i}:=\left[\begin{array}{lll}F_{i}^{(1)} & \ldots & F_{i}^{(L)}\end{array}\right]^{\mathrm{T}}, \forall i \in \mathcal{I}$ and the allocated time vector for downlink WET $\tau_{0}$ in matrix form as $\boldsymbol{F}_{i}=\Psi_{i} \boldsymbol{\tau}_{0}, \forall i \in \mathcal{I}$, where the $j$ th row and $k$ th element of $\Psi_{i} \in \mathbb{R}^{L \times L}$ is

$$
\Psi_{i}(\ell, j)= \begin{cases}p_{i, j}^{(\ell)} \beta_{i}^{(j)} & , 0 \leq j \leq \ell \\ 0 & , \ell<j \leq L\end{cases}
$$

and

$$
p_{i, j}^{(\ell)}=\alpha_{i}^{(\ell)} \prod_{k=j}^{\ell-1}\left(1-\alpha_{i}^{(k)}\right), \forall i \in \mathcal{I}, \forall j \in \mathcal{Q}_{\ell}, \forall \ell \in \mathcal{J}
$$

where $\mathcal{Q}_{\ell}:=\{1, \cdots, \ell\}$ is the set of the transmission block indices smaller than index $\ell$. If we define parameters $\phi_{i, j}^{(\ell)}:=$ $\gamma_{i}^{(\ell)} \beta_{i}^{(j)} p_{i, j}^{(\ell)}, \forall i \in \mathcal{I}, \forall j \in \mathcal{Q}_{\ell}, \forall \ell \in \mathcal{J}$, then we obtain the concrete expression of the throughput of user $U_{i}$ during $\ell$ th transmission block as

$$
R_{i}^{(\ell)}=\tau_{i}^{(\ell)} \log _{2}\left(1+\sum_{j=1}^{\ell} \phi_{i, j}^{(\ell)} \frac{\tau_{0}^{(j)}}{\tau_{i}^{(\ell)}}\right), \forall i \in \mathcal{I}, \forall \ell \in \mathcal{J} .
$$


If we plug (10) into the KKT conditions, then from the stationarity with respect to $\tau_{0}$, we have

$$
\sum_{\ell=j}^{L} \sum_{i=1}^{K}\left(\frac{\phi_{i, j}^{(\ell)}}{1+C_{i}^{(\ell) \star}}\right)=-\left(\nu^{(j) \star}+\lambda_{0}^{(j) \star}\right) \ln 2 .
$$

Similarly, from the stationarity with respect to $\tau$, it follows

$$
f\left(C_{i}^{(\ell) \star}\right)=-\left(\nu^{(\ell) \star}+\lambda_{i}^{(\ell) \star}\right) \ln 2, \forall i \in \mathcal{I}, \forall \ell \in \mathcal{J}
$$

where

$$
C_{i}^{(\ell) \star} \tau_{i}^{(\ell) \star}=\sum_{j=1}^{\ell} \phi_{i, j}^{(\ell)} \tau_{0}^{(j) \star}, \forall i \in \mathcal{I}, \forall \ell \in \mathcal{J}
$$

and

$$
f(x)=\ln (1+x)-\frac{x}{1+x} .
$$

From the equations obtained from the KKT conditions, the optimal time allocation vector of uplink WIT $\tau^{\star}$ can be uniquely determined by the optimal time allocation vector of downlink WET $\boldsymbol{\tau}_{0}^{\star}$, which is summarized in Theorem 1:

Theorem 1. The optimal time allocation vector of downlink WET $\tau_{0}^{\star}$ and the optimal time allocation vector of uplink WIT $\boldsymbol{\tau}^{\star}$ of $\boldsymbol{P} 3$ satisfy:

$$
\tau_{i}^{(\ell) \star}=\frac{\left(1-\tau_{0}^{(\ell) \star}\right) \sum_{j=1}^{\ell} \phi_{i, j}^{(\ell)} \tau_{0}^{(j) \star}}{\sum_{i=1}^{K} \sum_{j=1}^{\ell} \phi_{i, j}^{(\ell)} \tau_{0}^{(j) \star}}, \forall i \in \mathcal{I}, \forall \ell \in \mathcal{J} .
$$

The relation in (14) mainly comes from (12) and (13) and also the unit time block constraint. The details of the proof are omitted due to the space limitation. Since for any energy-consumed proportion vector $\boldsymbol{\alpha}$, (14) always holds. This indicates that we can first obtain a function of $\left(\boldsymbol{\tau}_{0}, \boldsymbol{\alpha}\right)$ by replacing $\boldsymbol{\tau}$ in the objective function $R\left(\boldsymbol{\tau}_{0}, \boldsymbol{\tau}, \boldsymbol{\alpha}\right)$ of $\mathbf{P} 2$ by using the relations in (14). The new objective function, which is denoted as $G\left(\boldsymbol{\tau}_{0}, \boldsymbol{\alpha}\right)$, can be expressed as

$$
\begin{aligned}
& G\left(\boldsymbol{\tau}_{0}, \boldsymbol{\alpha}\right) \\
& =\sum_{\ell=1}^{L}\left(1-\tau_{0}^{(\ell)}\right) \log _{2}\left(1+\frac{\sum_{i=1}^{K} \sum_{j=1}^{\ell} \phi_{i, j}^{(\ell)} \tau_{0}^{(j)}}{1-\tau_{0}^{(\ell)}}\right) .
\end{aligned}
$$

Mathematically, the optimization problem can be formulated in $\mathbf{P 4}$.

\section{Problem 4 (P4).}

$$
\begin{array}{ll}
\max _{\left(\boldsymbol{\tau}_{0}, \boldsymbol{\alpha}\right)} & G\left(\boldsymbol{\tau}_{0}, \boldsymbol{\alpha}\right) \\
\text { s.t. } & \mathbf{0} \preceq \boldsymbol{\tau}_{0} \preceq \mathbf{1} \\
& \mathbf{0} \preceq \boldsymbol{\alpha} \preceq \mathbf{1}
\end{array}
$$

If we concatenate the vector $\boldsymbol{\alpha}$ to the vector $\boldsymbol{\tau}_{0}$ to form a larger vector $\boldsymbol{\delta} \in \mathbb{R}^{(K+1) \times 1}$, i.e., $\boldsymbol{\delta}=\left[\begin{array}{ll}\boldsymbol{\tau}_{0}^{\mathrm{T}} & \boldsymbol{\alpha}^{\mathrm{T}}\end{array}\right]^{\mathrm{T}}$, then, $\mathbf{P 4}$ is equivalent to the problem of finding the vector $\delta$ to maximize $G(\boldsymbol{\delta})$ subject to $\mathbf{0} \preceq \boldsymbol{\delta} \preceq \mathbf{1}$. This problem is a standard boxconstrained optimization problem, which can be solved using the trust-region-reflective algorithm [16], [17].

\section{UPPER BOUND}

Although the algorithm in Section III gives us the optimal solution of P1, the computational complexity is high when the number of users $K$ or the number of transmission blocks $L$ grows. In this section, we provide an upper bound of the optimal sum throughput in $\mathbf{P 1}$ by relaxing the energy harvesting causality, which gives us a water-filling solution.

In P1, we assume that the energy causality condition holds, i.e., the amount of energy consumed in each block can not exceed the current energy stored at each user, which is shown in (3). Now we reconsider the optimization problem with the constraint that the total consumed energy does not exceed the total harvested energy at each user, i.e., $\sum_{j=1}^{L} F_{i}^{(j)} \leq$ $\sum_{j=1}^{L} E_{i}^{(j)}, \forall i \in \mathcal{I}$. If we plug the relation $\gamma_{i}^{(\ell)} F_{i}^{(\ell)}=$ $\sum_{j=1}^{\ell} \phi_{i, j}^{(\ell)} \tau_{0}^{(j)}, \forall i \in \mathcal{I}, \forall \ell \in \mathcal{J}$ into (15) and construct the consumed energy vector $\boldsymbol{F}$ by replacing the element $\alpha_{i}^{(\ell)}$ with $F_{i}^{(\ell)}$ in $\boldsymbol{\alpha}$, then, the new objective function can be expressed as

$$
T\left(\boldsymbol{\tau}_{0}, \boldsymbol{F}\right)=\sum_{\ell=1}^{L}\left(1-\tau_{0}^{(\ell)}\right) \log _{2}\left(1+\frac{\sum_{i=1}^{K} \gamma_{i}^{(\ell)} F_{i}^{(\ell)}}{1-\tau_{0}^{(\ell)}}\right) .
$$

Thus, the corresponding optimization problem can be formulated as

Problem 5 (P5).

$$
\begin{array}{ll}
\max _{\left(\boldsymbol{\tau}_{0}, \boldsymbol{F}\right)} & T\left(\boldsymbol{\tau}_{0}, \boldsymbol{F}\right) \\
\text { s.t. } & \mathbf{0} \preceq \boldsymbol{\tau}_{0} \preceq \mathbf{1} \\
& \mathbf{0} \preceq \boldsymbol{F} \\
& \sum_{\ell=1}^{L} F_{i}^{(\ell)} \leq \sum_{\ell=1}^{L} E_{i}^{(\ell)}, \forall i \in \mathcal{I} .
\end{array}
$$

Observe that $\mathbf{P 5}$ relaxes $(L-1) K$ conditions in P1. Thus the maximum sum throughput in $\mathbf{P 5}$ gives us an upper bound of that in P1. To solve P5, we can first fix $\boldsymbol{\tau}_{0}$ and find the optimal solution of $\boldsymbol{F}$. If we define a new vector $\tilde{\boldsymbol{F}}:=\left[\begin{array}{lll}\tilde{F}^{(1)} & \cdots & \tilde{F}^{(L)}\end{array}\right]^{\mathrm{T}}$ with $\tilde{F}^{(\ell)}=\sum_{i=1}^{K} \gamma_{i}^{(\ell)} F_{i}^{(\ell)}, \forall i \in$ $\mathcal{I}, \forall \ell \in \mathcal{J}$, we can obtain the optimal solution.

Theorem 2. The optimal solution of $\tilde{\boldsymbol{F}}$ when fixing $\boldsymbol{\tau}_{0}$ is

$$
\tilde{F}^{(\ell)}=\left[\frac{1-\tau_{0}^{(\ell)}}{\pi^{\star}}-\left(1-\tau_{0}^{(\ell)}\right)\right]^{+}, \forall \ell \in \mathcal{J}
$$

where $\pi^{\star} \in \mathbb{R}$ is selected to satisfy

$$
\sum_{\ell=1}^{L} \tilde{F}^{(\ell)}=\sum_{\ell=1}^{L} \sum_{i=1}^{K} \gamma_{i}^{(\ell)} E_{i}^{(\ell)} .
$$

The proof of Theorem 2 is mainly obtained by using KKT conditions. The details are omitted due to the space limitation.

After solving $\tilde{\boldsymbol{F}}$, we notice that the problem of finding optimal $\tau_{0}$ is a standard box-constrained nonlinear programming problem like $\mathbf{P 4}$, which can be solved efficiently using the trust-region-reflective algorithm [16], [17]. 


\section{Numerical Results}

In this section, we compare the maximum sum throughput using energy saving with systems in which the users are assumed to use all their energy within current block. We continue to use the simulation parameters in [15]. The bandwidth is assumed to be $1 \mathrm{MHz}$. Both the downlink and uplink channel power gains are modeled as $h_{i}^{(\ell)}=g_{i}^{(\ell)}=10^{-3} \rho_{i}^{(\ell) 2} D_{i}^{-\theta}, i=$ $1, \cdots, K ; \ell=1, \cdots, L$, where $\theta=2$ is the pathloss exponent and $\rho_{i}^{(\ell)}$ represents the channel short-term fading of the $i$ th channel within the $\ell$ th block. The short-term fading is assumed to be Rayleigh distributed, hence $\rho_{i}^{(\ell) 2}$ is an exponentially distributed random variable with unit mean. The $K$ users in the network are equally separated from the H-AP according to $D_{i}=\frac{D_{K}}{K} \times i, i=1, \cdots, K$, where $D_{K}=10 \mathrm{~m}$. The AWGN at the $\mathrm{H}$-AP receiver is assumed to have a white power spectral density of $-160 \mathrm{dBm} / \mathrm{Hz}$. For each user, the energy harvesting efficiency for WET is assumed to be $\zeta=0.5$. We assume that an uncoded quadrature amplitude modulation (QAM) is employed and thus $\Gamma=9.8 \mathrm{~dB}$.

Fig. 3 shows the normalized maximum sum throughput versus the number of transmission blocks for different number of users. As shown in Fig. 3, the normalized sum throughput increases when the number of transmission blocks grows. The numbers in the figure shows the possible maximum percentage gain by using energy saving, i.e., $15 \%$ for $K=3,13 \%$ for $K=4$ and $12 \%$ for $K=5$. It is observed that when number of users grows, the possible maximum percentage gain will decrease. The black dashed curve shows the upper bound of the maximum sum throughput. It is observed that the upper bound of the maximum sum throughput will be close to the actual maximum sum throughput when $K$ is large.

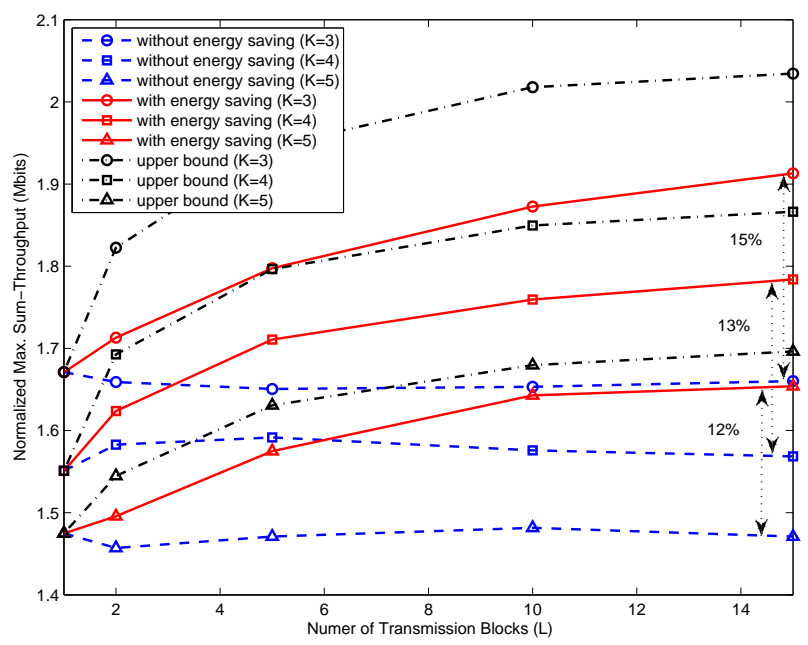

Fig. 3. Normalized maximum sum throughput vs. number of transmission blocks $(L)$.

\section{CONCLUSION}

In this paper, we have studied the throughput maximization problem in WPCN with a finite-horizon energy saving scheme.
To obtain the optimal solution, the initial optimization problem is separated into two sub-problems and finally is formulated into a standard box-constrained optimization problem, which can be solved efficiently by the trust-region-reflective algorithm. We have observed that the improvement of the sum throughput with long-term energy saving is not considerable if considering the "oracle" and the computational complexity. This indicates that the initial scheme without energy saving is a practical and favorable strategy in WPCN [15].

\section{REFERENCES}

[1] A. Ephremides, "Energy concerns in wireless networks," Wireless Communications, IEEE Transactions on, vol. 9, no. 4, pp. 48-59, Aug 2002.

[2] J. A. Paradiso and T. Starner, "Energy scavenging for mobile and wireless electronics," IEEE Pervasive Computing, vol. 4, no. 1, pp. 1827, 2005.

[3] S. Sudevalayam and P. Kulkarni, "Energy harvesting sensor nodes: Survey and implications," Communications Surveys Tutorials, IEEE, vol. 13, no. 3, pp. 443-461, Mar 2011.

[4] V. Liu, A. Parks, V. Talla, S. Gollakota, D. Wetherall, and J. R. Smith, "Ambient backscatter: Wireless communication out of thin air," in Proceedings of the ACM SIGCOMM 2013 Conference on SIGCOMM, 2013, pp. 39-50.

[5] K. Finkenzeller, RFID Handbook: Fundamentals and Applications in Contactless Smart Cards and Identification, 2003.

[6] J. R. Smith, Wirelessly Powered Sensor Networks and Computational RFID. Springer, 2013.

[7] L. Varshney, "Transporting information and energy simultaneously," in Information Theory, 2008. ISIT 2008. IEEE International Symposium on, July 2008, pp. 1612-1616.

[8] X. Zhou, R. Zhang, and C. K. Ho, "Wireless information and power transfer: Architecture design and rate-energy tradeoff," Communications, IEEE Transactions on, vol. 61, no. 11, pp. 4754-4767, November 2013.

[9] L. Liu, R. Zhang, and K.-C. Chua, "Wireless information and power transfer: A dynamic power splitting approach," Communications, IEEE Transactions on, vol. 61, no. 9, pp. 3990-4001, September 2013.

[10] P. Grover and A. Sahai, "Shannon meets tesla: Wireless information and power transfer," in Information Theory Proceedings (ISIT), 2010 IEEE International Symposium on, June 2010, pp. 2363-2367.

[11] R. Zhang and C. K. Ho, "Mimo broadcasting for simultaneous wireless information and power transfer," Wireless Communications, IEEE Transactions on, vol. 12, no. 5, pp. 1989-2001, May 2013.

[12] A. Fu, E. Modiano, and J. Tsitsiklis, "Optimal transmission scheduling over a fading channel with energy and deadline constraints," Wireless Communications, IEEE Transactions on, vol. 5, no. 3, pp. 630-641, March 2006.

[13] N. Michelusi, K. Stamatiou, and M. Zorzi, "On optimal transmission policies for energy harvesting devices," in Information Theory and Applications Workshop (ITA), 2012, Feb 2012, pp. 249-254.

[14] R. Vaze and K. Jagannathan, "Finite-horizon optimal transmission policies for energy harvesting sensors," in Acoustics, Speech and Signal Processing (ICASSP), 2014 IEEE International Conference on, May 2014, pp. 3518-3522.

[15] H. Ju and R. Zhang, "Throughput maximization in wireless powered communication networks," Wireless Communications, IEEE Transactions on, vol. 13, no. 1, pp. 418-428, January 2014.

[16] T. F. Coleman and Y. Li, "An interior trust region approach for nonlinear minimization subject to bounds," SIAM Journal on Optimization, vol. 6, no. 2, pp. 418-445, 1996.

[17] _ _ "On the convergence of reflective newton methods for large-scale nonlinear minimization subject to bounds," Mathematical Programming, vol. 67, no. 2, pp. 189-224, 1994.

[18] S. Boyd and L. Vandenberghe, Convex Optimization. Cambridge University Press, 2004. 